\title{
Leven, Eva-Maria (2019). Professionalität von Religionslehrkräften. Eine explorative Studie zur Rekonstruktion fachspezifischen Professionswissens sowie handlungsbezogener und reflexiver Kompetenzen von Religionslehrkräften (Empirische Theologie/Empirical Theology). Münster: Lit. ISBN 978-3-643-| 4266-5. 5 I 3 Seiten.
}

\section{Carina Caruso}

Universität Paderborn (ccaruso@mail.uni-paderborn.de)

Die Begriffe professionell und Professionalität finden in verschiedenen Kontexten Verwendung. Dies erschwert die Antwort auf die Frage danach, was professionelles Handeln von Lehrpersonen kennzeichnet. Im religionspädagogischen Bereich werden häufig die Lehrerpersönlichkeit sowie Überzeugungen von Religionslehrpersonen als wichtige Stellgrößen für die Professionalität herausgearbeitet (28-34). Auch in fachübergreifenden Arbeiten sind dies wichtige Komponenten von Lehrerprofessionalität. Gleichzeitig ist jedoch in Anlehnung an bildungswissenschaftliche Diskurse, die die Lehrerprofessionalität und -professionalisierung aus kompetenzorientierter Perspektive (13-15) thematisieren, davon auszugehen, dass das Professionswissen von Lehrpersonen einen wichtigen Ausgangspunkt für ihr professionelles Handeln markiert.

Dies erklärt, warum sich in bildungswissenschaftlichen und fachdidaktischen Diskursen u. a. in Bereichen des Professionswissens Strukturmodelle finden, die empirisch auf den Prüfstand gestellt werden. In der Religionspädagogik hingegen sind Arbeiten, die das Professionswissen oder das professionelle Lehrerhandeln fokussieren und empirisch erfassen, vergleichsweise selten.

Leven nimmt sich diesem Forschungsdesiderat an und legt eine religionsdidaktische Arbeit vor, die zeigt, wie religionspädagogische Fragestellungen systematisch und gehaltvoll mit Perspektiven der Bildungswissenschaften und denen anderer Fachdidaktiken verknüpft werden können. Sie entwirft ein Strukturmodell zum Professionswissen von Religionslehrpersonen mit dem Ziel, dies empirisch zu sättigen und untersucht am Umgang mit religiösen Wahrheitsfragen domänenspezifische Merkmale professionellen Religionslehrerhandelns im Rahmen der Vorbereitung, Durchführung und Nachbereitung von Religionsunterricht. Dabei geht Leven davon aus, dass sich Professionswissen in der Phase der Unterrichtsvorbereitung erfassen lässt, wohingegen die Unterrichtsdurchführung herangezogen werden kann, um handlungsbezogene Kompetenzen zu eruieren. Reflexive Kompetenzen sind hingegen solche, die in der Nachbereitung von Unterricht zum Tragen kommen.

Der erste Teil der Arbeit konstituiert sich aus drei Einheiten: Lehrkräfte als professionell Handelnde, Professionalität an einem Beispiel und Gesamtdesign der Studie. Leven bezieht sich zu Beginn auf Bestimmungsansätze lehrerspezifischer Professionalität, beschreibt ein Strukturmodell professionellen Handelns und thematisiert die Professionalität von Religionslehrpersonen. Begriffe werden dabei systematisch eingeführt.

Im ersten Teil benennt sie daran anschließend die grundlegende Fragestellung der Arbeit: Wie äußert sich fachspezifische Professionalität von Religionslehrkräften in der Planung, Durchführung und Nachbereitung von Religionsunterricht und inwiefern lässt sich dies mithilfe bestehender Konzepte zum professionellen Handeln von Lehrkräften beschreiben? (35) Ihr Zugang zur Beantwortung der Forschungsfrage besteht aus Interviews, Videoaufzeichnungen und Video-stimulated Recalls. Aus dieser Fragestellung ergibt sich, dass Leven in ihre Arbeit zwei Untersuchungsabschnitte integriert (Teil II und Teil III). In Teil II fokussiert 
sie das Professionswissen von Religionslehrpersonen. Dabei beantwortet sie die folgende Forschungsfrage: Welche Form und Struktur weist das Professionswissen der untersuchten Religionslehrkräfte in der Planung von Religionsunterricht auf und lässt sich das fachspezifische Wissen mit den bekannten Wissensformen und -strukturen aus dem heuristischen Modell beschreiben? (36) Teil III widmet sich der Exploration einer pädagogischen Gesamthandlung in der Planung, Durchführung und Nachbereitung von Fachunterricht (36). Daraus ergibt sich eine weitere Forschungsfrage, die dreigliedrig ist: Welche Vorstellungen äußern Religionslehrpersonen bezüglich des Umgangs mit religiösen Glaubenswahrheiten im Planungsinterview? Wie gelingt den Lehrkräften der Umgang mit Wahrheitsfragen im Unterricht? Was und wie reflektieren die Lehrpersonen bezüglich des Umgangs mit Wahrheitsfragen im Unterricht? (36)

Nachdem im ersten Teil der Arbeit die einzelnen Fragestellungen eingeführt werden, thematisiert Leven die Professionalität am Beispiel des Themas Jesu Christus. Sie verweist darauf, dass sie nur exemplarisch rekonstruieren kann, was ein professionelles Handeln von Lehrpersonen prägt, da jeder Inhalt, der im Unterricht bearbeitet wird, unterschiedliches Fachwissen und didaktisches Wissen fordert. Der erste Teil der Arbeit schließt mit Ausführungen zum Gesamtdesign. Präzise skizziert Leven dabei die Rahmenbedingungen der Studie, den Aufbau der Untersuchungen und erläutert die Untersuchungsmethoden, die den Untersuchungsabschnitten zugeordnet sind. Um die Professionalität von Religionslehrkräften explorieren zu können, „,begleitet die Studie drei Religionslehrkräfte für die Realschule über die drei Phasen einer gesamten fachunterrichtlichen Handlung - Unterrichtsvorbereitung, -durchführung und -nachbereitung mit verschiedenen qualitativen Methoden“ (89). Die Lehrkräfte unterrichten Jesus Christus.

Im Zentrum von Teil II steht das Professionswissen. Ein Strukturmodell theologischen und religionsdidaktischen Wissens bildet das Fundament des Leitfadens problemzentrierter Interviews. Diese werden in der Phase der Unterrichtsvorbereitung geführt und inhaltsanalytisch ausgewertet, was Leven erlaubt, ein empirisch gesättigtes Strukturmodell zu entwerfen. Die Ergebnisse werden systematisch entlang der Kategorien beschrieben.

In Teil III wird das professionelle Handeln rekonstruiert. Dabei bedient sich Leven eines triangulativen Verfahrens. Daten der Planungsinterviews, Videodaten aus Unterrichtsaufzeichnungen und Kommentare aus der Nachbereitung werden miteinander in Beziehung gesetzt. Zur Erhebung des Planungswissens zum Umgang mit Wahrheitsfragen werden die Interviews aus Teil II erneut ausgewertet. Die aufgezeichneten Unterrichtsreihen dienen der Beschreibung handlungsbezogener Kompetenzen, die mit einem hoch-inferenten Rating und der Dokumentarischen Methode ausgewertet werden. „Zur Analyse der reflexiven Fähigkeiten werden im Anschluss an die Unterrichtsreihen Video-stimulated Recalls durchgeführt, die ebenfalls mit der Dokumentarischen Methode ausgewertet werden“ (5). Leven beschreibt die Professionalität somit ausgehend von der Praxis beruflichen Handelns. In Bezug auf Wissen, Können und Reflexion werden die Ergebnisse beschrieben und anschließend personenbezogen ausgewertet.

Teil IV widmet sich der Diskussion der Befunde und der Methodik. Dabei werden die Befunde vor dem Hintergrund der theoretischen Ausführungen angemessen diskutiert sowie Limitationen und anschließende Forschungsdesiderate aufgeführt.

Die Arbeit ist insgesamt stringent aufgebaut und liefert wichtige Erkenntnisse zur Professionalität von Religionslehrkräften. Begriffe werden konsequent eingeführt und das methodische Vorgehen erläutert und reflektiert. Trotz ihrer Länge ist die Arbeit verständlich, was u. a. dadurch unterstützt wird, dass dem ersten Teil der Arbeit eine Einleitung voransteht, die den Aufbau der Arbeit vorstellt, in einzelne Kapitel eingeführt wird und diese jeweils mit einem kurzen Fazit schließen. Die auf den ersten Blick unübersichtliche formale Gliederung der Arbeit (Teile, Buchstaben, Nummerierung) sowie die Feststellung, dass die Integration von einigen Abbildungen, um die Inhalte zu illustrieren, wünschenswert gewesen wäre, trüben den guten Eindruck der Arbeit nicht. 